JOURNAL OF INTEGRAL EQUATIONS

AND APPLICATIONS

Volume 18, Number 3, Fall 2006

\title{
PROLATE SPHEROIDAL WAVELETS IN HIGHER DIMENSIONS
}

\author{
GILBERT G. WALTER
}

\begin{abstract}
Prolate spheroidal wavelets (PS wavelets) based on the first prolate spheroidal wave function (PSWF), were recently introduced. They were shown to have many desirable properties lacking in other wavelets. In particular, the subspaces belonging to the associated MRA were shown to be closed under differentiation and translation. In this paper, we introduce prolate spheroidal wavelets in higher dimensions. They are similar to the one-dimensional versions in that they are based on an eigenfunction of an integral operator. But there is not, in general, an associated differential operator which is helpful in the one dimensional case for construction. Hence another method of construction must be used.
\end{abstract}

1. Introduction. The prolate spheroidal wave functions (PSWFs), $\left\{\varphi_{n, \sigma, \tau}(t)\right\}_{n \in Z}$, constitute an orthonormal basis of the space of $\sigma$ bandlimited functions on the real line. They are the eigenfunctions of an integral operator with the sinc function, $S(t)=\sin \pi t / \pi t$, as its kernel:

$$
\int_{-\tau}^{\tau} \varphi_{n, \sigma, \tau}(x) \frac{1}{T} S\left(\frac{t-x}{T}\right) d x=\lambda_{n, \sigma, \tau} \varphi_{n, \sigma, \tau}(t)
$$

where $T=\pi / \sigma$. They were obtained, in a series of papers by Slepian, Pollak and Landau at Bell Labs, as the solutions of an energy concentration problem which led to this integral equation. The problem was to find the normalized $\sigma$-bandlimited function with the maximum energy concentration on the interval $[-\tau, \tau]$. The solution is the first PSWF $\varphi_{0, \sigma, \tau}$; the function orthogonal to $\varphi_{0, \sigma, \tau}$ which possesses the maximum energy concentration on $[-\tau, \tau]$ is $\varphi_{1, \sigma, \tau}$, etc. They are also solutions to a Sturm-Liouville problem arising from the Helmholz equation on the prolate spheroid. Ergo the name.

Key words and phrases. Prolate spheroidal wave functions, wavelets, PS wavelets, bandlimited signal, Paley-Wiener space.

Received by the editors on May 3, 2005, and in revised form on December 27, 2005.

Copyright (C)2006 Rocky Mountain Mathematics Consortium 
The system carries two parameters, $\sigma$ and $\tau$, either one of which can be eliminated by a change of variable. The properties of these PSWFs were extensively studied and reported in the classic papers $[\mathbf{2}, \mathbf{3}, \mathbf{4}, \mathbf{7}$, 8, 9] during the 1960's.

Recently, there has been renewed interest in PSWFs in part because of their sampling [15] and multi-scale properties [14]. A system of wavelets based on these PSWFs, made possible by their multi-scale nature, was introduced in an earlier paper [14] (called PS wavelets for short). The PS wavelets were constructed in such a way as to retain the energy concentration property and were shown to have many desirable properties lacking in other wavelet systems. Just as with the PSWFs, these wavelets are entire functions with finite energy, but by choosing $\tau$ appropriately, can be made arbitrarily small outside of $[-\tau, \tau]$.

We organize this paper as follows. This section is followed by Section 2, in which we recall some related properties of PS wavelets in one dimension. This is followed by consideration of the maximization problem in higher dimensions with a discussion of the associated eigenvalue problem. The system of wavelets follows as in one dimension for appropriate regions $\Omega$, and has similar properties. Unfortunately, the "lucky accident" in which the PSWFs are simultaneously eigenfunctions of a differential operator and an integral operator fails to hold in general in higher dimensions but can be replaced by another remarkable accident. In special cases such as when $\Omega$ consists of a disk, associated differential operators can be found, but these are not the regions that lead to PS wavelets. The disk case was already studied by Slepian who developed it in detail [8].

2. PS wavelets in one dimension. Before constructing the higher dimensional PS wavelets, we review the PS wavelets in one dimension introduced in [14]. A scaling function $\phi=\varphi_{0, \pi, \tau}$ was defined as the $\pi$ bandlimited function of norm 1 whose energy on $[-\tau, \tau]$ is maximized. The integer translates formed a Riesz basis of the space $V_{0} \subset L^{2}(\mathbf{R})$. This space $V_{0}$ turned out to be the Paley-Wiener space $B_{\pi}$ of $\pi$ bandlimited functions no matter what the choice of $\tau>0$.

This space then becomes part of the family of nested subspaces $\left\{V_{m}\right\}$ of a multi-resolution analysis (MRA). The other spaces are obtained, as usual, by dilations by factors of two and consist of the Paley-Wiener 
spaces $V_{m}=B_{2^{m} \pi}$. Because they are entire functions, the bandlimited functions cannot have compact support in the time domain. The maximization problem, more precisely, is that of maximizing

$$
\alpha(h, \tau) \equiv\left(\int_{-\tau}^{\tau}\left|h^{2}(t)\right| d t\right)^{1 / 2}, \quad h \in B_{\pi},\|h\|_{L^{2}(\mathbf{R})}=1 .
$$

and is solved by $\phi=\varphi_{0, \pi, \tau}$. Although $\alpha$ can never be equal to 1 , for $\tau$ sufficiently large the energy of $\phi$ can be made arbitrarily small outside of the interval of concentration $[-\tau, \tau]$. For example, for $\tau=2, \sigma=\pi$, the total energy outside of the interval is of the order of $10^{-6}$. Hence the PS wavelets are not only superior as far as analytic properties are concerned, but also similar to compactly supported wavelets for most practical computations.

We recall the following properties proved in $[\mathbf{1 3}, \mathbf{1 4}]$.

Proposition 1. Let $\phi(t)=\varphi_{0, \pi, \tau}(t)$ be a $\pi$-bandlimited PSWF with concentration interval $[-\tau, \tau]$; then $\{\phi(t-k)\}$ is a Riesz basis of $B_{\pi}$. The PS mother wavelet, in turn, is given by

$$
\psi(t):=\cos \left(\frac{3 \pi}{2} t\right) \varphi_{0, \pi / 2, \tau / 2}(t),
$$

which is orthogonal to all integer translates of $\phi(t)$. The translates of the mother wavelet form a Riesz basis of the orthogonal complement of $V_{0}$ in $V_{1}$.

As usual, we denote the wavelet subspace by $W_{0}=\overline{\operatorname{span}\{\psi(t-n)\}}$ with its dilations denoted by $W_{m}=\overline{\operatorname{span}\left\{2^{m / 2} \psi\left(2^{m} t-k\right)\right\}}, m \in \mathbf{Z}$.

In most of the standard wavelets, the derivatives of the scaling function do not belong to the space $V_{0}$, nor for that matter, to any of the subspaces $V_{m}$. The exceptions are the Meyer wavelets which do not have compact support, but even in this case the derivatives do not belong to $V_{0}$. Furthermore, none of the scaling functions with compact support belong to $C^{\infty}$, so that they cannot be differentiated arbitrarily often $[\mathbf{1}]$.

For any wavelet system the translation of an $f \in V_{0}$ by an integer is again in $V_{0}$, but if it is translated by some other real number, it no 
longer belongs to $V_{0}$. In fact, in most cases, it no longer belongs to any of the subspaces $V_{m}$. This, of course, becomes a problem when there are measurements based on an independent variable without a natural zero, for example if the independent variable is time. In fact, we have the following $[\mathbf{1 3}]$ :

Proposition 2. Let $\phi(t)=\varphi_{0, \pi, \tau}(t)$ be the PS scaling function. Then

(i) $\phi^{(k)} \in V_{0}$, for $k=0,1, \ldots$;

(ii) $\phi(t-\beta) \in V_{0}$ for any $\beta \in \mathbf{R}$; and

(iii) if $f \in V_{0}$ has the expansion

$$
f(t)=\sum_{n=-\infty}^{\infty} \alpha_{n} \phi(t-n)
$$

then $f^{\prime} \in V_{0}$ and

$$
f^{\prime}(t)=\sum_{n=-\infty}^{\infty} a_{n} \phi(t-n)
$$

where $a_{n}=\sum_{j \neq n}\left((-1)^{j-n} /(j-n)\right) \alpha_{j}$;

(iv) moreover, if $\beta$ is not an integer, then

$$
f(t-\beta)=\sum_{n=-\infty}^{\infty} b_{n} \phi(t-n),
$$

where $b_{n}=\sum_{j=-\infty}^{\infty} \alpha_{j} S(n-j-\beta)$.

Formula (4) indicates that differentiation can be reduced to a simple algebraic operation (discrete convolution) just as with Fourier series. The same is true for translations. Other operations such as dilation and convolution were also shown to have simple expressions in terms of the coefficients. 
3. Maximization in higher dimensions. The scaling function of the PS wavelets was defined as the first PSWF $\varphi_{0, \pi, \tau}(t)$, the one with maximum concentration on $[-\tau, \tau]$ among normalized $\pi$-bandlimited functions. In $\mathbf{R}^{d}$ in general, we replace the bandlimiting interval $[-\sigma, \sigma]$ in the Fourier transform domain by an arbitrary compact set $\Omega \subset \mathbf{R}^{d}$. In the time domain, we replace the concentration interval $[-\tau, \tau]$ by another compact set $\Gamma \subset \mathbf{R}^{d}$ To avoid pathology, we shall assume that both sets have positive measure. We denote by $\mathcal{B}_{\Omega}$ the subspace of $L^{2}\left(\mathbf{R}^{d}\right)$ composed of functions which are the inverse Fourier transform of a function with compact support in $\Omega$. The maximization problem (2) is replaced by the problem of finding the function which maximizes $\alpha$ given by

$$
\alpha(h, \Gamma) \equiv\left(\int_{\Gamma}\left|h^{2}(t)\right| d t\right)^{1 / 2}, \quad h \in B_{\Omega},\|h\|_{L^{2}\left(\mathbf{R}^{d}\right)}=1 .
$$

The solution to this problem will be the multi-dimensional analog of the first PS wave function $\varphi_{0, \sigma, \tau}(t)$. Since this is all we need to construct the scaling function, we shall not bother with the other PS wave functions. Later we will specialize our choice of $\Omega$ to make possible a wavelet basis.

This is the same maximization problem considered by Slepian in [8]; his results for the disk are comparable to the one-dimensional case and give a complete set of prolate spheroidal wave functions.

3.1 An eigenvalue problem. In the one-dimensional case the maximization problem is solved by converting it to an eigenvalue problem for an integral operator. This operator, in turn, is shown to commute with a differential operator which therefore has the same eigenfunctions. Because of this "lucky accident" the differential operator can be used for calculation. In higher dimensions, the differential operator is usually not available (except in the case of a ball), but instead a new method of calculating the eigenfunctions based directly on the integral operator is used. This procedure is a generalization of the one-dimensional procedure introduced in $[\mathbf{1 0}]$.

The following proposition is a straightforward generalization of the result in one dimension and is given in $[\mathbf{8}]$ with a slightly different approach. 
Proposition 3. Let $\Omega, \Gamma$ be compact sets in $\mathbf{R}^{d}$ of positive measure, let $S_{\Omega}(t)$ be the inverse Fourier transform of $\chi_{\Omega}$, the characteristic function of $\Omega$; then the solution to the maximization problem (6) is given by the solution to the eigenvalue problem

$$
\int_{\Gamma} S_{\Omega}(t-x) f(x) d x=\lambda f(t)
$$

with the largest eigenvalue.

The space $\mathcal{B}_{\Omega}$ is just the multi-dimensional version of a Paley-Wiener space. Let $f \in \mathcal{B}_{\Omega}$; then the Fourier transform satisfies $\widehat{f}=\widehat{f} \cdot \chi_{\Omega}$ since $f$ has support in $\Omega$. Then, by taking the inverse Fourier transform, and using the fact that convolution transforms into multiplication, we find that

$$
f=f * S_{\Omega} .
$$

That is, the function $S_{\Omega}(t-x)$ is just the reproducing kernel of $\mathcal{B}_{\Omega}$.

If we now assume that $f$ has unit norm in the $L^{2}\left(\mathbf{R}^{d}\right)$ sense, then the maximization problem becomes one of maximizing

$$
\int_{\Gamma}\left|f * S_{\Omega}(t)\right|^{2} d t=\left\langle S_{\Omega, \Gamma} f, S_{\Omega, \Gamma} f\right\rangle_{\Gamma}=\left\|S_{\Omega, \Gamma} f\right\|_{\Gamma}^{2}
$$

where $S_{\Omega, \Gamma}$ is the operator on $\mathcal{B}_{\Omega}$ with kernel $S_{\Omega}(t-x) \chi_{\Gamma}(t)$. This same formula can also be expressed as

$$
\int_{\Gamma}\left|f * S_{\Omega}(t)\right|^{2} d t=\int_{\Gamma}\left(f * S_{\Omega}\right)(t) \overline{f(t)} d t=\left\langle S_{\Omega, \Gamma} f, f\right\rangle_{\Gamma}
$$

by the reproducing property of $S_{\Omega}(t-x)$. This shows us the operator is nonnegative and, in fact, is positive. This follows from the fact that if it were equal to zero for some nonzero $f \in \mathcal{B}_{\Omega}$, then $f$ would be zero on $\Gamma$, a distinct impossibility since $\mathcal{B}_{\Omega}$ is composed of entire functions in $\mathbf{C}^{d}$. A similar argument shows it to be self-adjoint. Furthermore, it is a Hilbert-Schmidt operator since

$$
\int_{\Gamma} \int_{\mathbf{R}^{d}}\left|S_{\Omega}(t-x)\right|^{2} d x d t=\int_{\Gamma} \int_{\mathbf{R}^{d}}\left|S_{\Omega}(x)\right|^{2} d x d t=\int_{\Gamma} 1 d t<\infty,
$$

i.e., $S_{\Omega}(t-x) \chi_{\Gamma}(t) \in L^{2}\left(\mathbf{R}^{2 d}\right)$. 
Hence there exists a sequence of positive real eigenvalues $\lambda_{0} \geq \lambda_{1} \geq$ $\lambda_{2} \geq \cdots>0$, with their corresponding normalized eigenfunctions $\varphi_{0, \Omega, \Gamma}, \varphi_{1, \Omega, \Gamma}, \varphi_{2, \Omega, \Gamma}, \ldots$ Thus the solution to the maximization problem is given by $f=\varphi_{0, \Omega, \Gamma}$, and $\lambda_{0}=\left\langle S_{\Omega, \Gamma} \varphi_{0, \Omega, \Gamma}, \varphi_{0, \Omega, \Gamma}\right\rangle$ is the maximum value.

In one dimension, the eigenvalues all have multiplicity one, but the proof of this uses the differential equation of the PSWF as well [9]. This is also true for very regular regions in higher dimensions, but in general, we can conclude only that each eigenvalue has finite multiplicity. These eigenfunctions $\left\{\varphi_{n, \Omega, \Gamma}\right\}$ constitute the $d$-dimensional PSFWs associated with the sets $\Omega$ and $\Gamma$; they are orthogonal on $\Gamma$, and, in fact, constitute an orthogonal basis of $L^{2}(\Gamma)$ since 0 is not an eigenvalue of such a positive operator. They are also orthogonal on $\mathbf{R}^{d}$ which can easily be shown as in one dimension.

We shall not pursue this orthonormal system further since we are mainly interested in the first function $\varphi_{0, \Omega, \Gamma}$ which we shall use to construct a wavelet system.

\section{Examples of Kernels.}

- The standard one-dimensional case is the symmetric interval $\Omega=$ $[-\sigma, \sigma]$, for which the kernel can be given in closed form $S_{\Omega}(t)=$ $(\sin \sigma t / \pi t)$. This extends immediately to a $d$-dimensional interval $\Omega=$ $[-\sigma, \sigma]^{d}$; the kernel is $S_{\Omega}\left(t_{1}, t_{2}, \ldots, t_{d}\right)=\left(\sin \sigma t_{1} / \pi t_{1}\right) \cdot\left(\sin \sigma t_{2} / \pi t_{2}\right.$. $\ldots \cdot\left(\sin \sigma t_{d} / \pi t_{d}\right)$.

- For the triangular region bounded by $\omega_{1}+\omega_{2}=\pi,-\omega_{1}+\omega_{2}=$ $\pi, \omega_{2}=0$, the kernel is $S_{\Omega}\left(t_{1}, t_{2}\right)=\left[2 t_{1} e^{i \pi t_{2}}-2\left(t_{1}+t_{2}\right) e^{i \pi t_{1}}+\right.$ $\left.2\left(t_{1}-t_{2}\right) e^{-i \pi t_{1}}\right] / 4 \pi^{2}\left(t_{1}^{2}-t_{2}^{2}\right) t_{1}$. Similarly for other regions in the plane bounded by straight lines.

- The ball in three dimensions $\omega_{1}^{2}+\omega_{2}^{2}+\omega_{3}^{2} \leq 1$ gives us $S_{\Omega}\left(t_{1}, t_{2}, t_{3}\right)=$ $1 /\left(2 \pi^{2}|t|^{3}\right)[\sin |t|-|t| \cos |t|]$ where $|t|=\left(t_{1}^{2}+t_{2}^{2}+t_{3}^{2}\right)^{1 / 2}$.

- The disk in two dimensions $\omega_{1}^{2}+\omega_{2}^{2} \leq 1$ leads to the kernel $S_{\Omega}\left(t_{1}, t_{2}\right)=1 /(2 \pi) \int_{0}^{1} J_{0}(|t| r) r d r=\left(J_{1}(|t|) / 2 \pi|t|\right)$ where $|t|=\left(t_{1}^{2}+\right.$ $\left.t_{2}^{2}\right)^{1 / 2}$ and $J_{0}, J_{1}$ are the Bessel functions of order 0 and 1 respectively, [6]. In this example, the PSWFs turn out to be given by eigenfunctions of the integral operator with the same Bessel function kernel [8]. 
3.2 Sampling function. Up to now our regions $\Omega$ and $\Gamma$ are quite general, but we need some restrictions in order to get a scaling function whose integer translates from a basis of $\mathcal{B}_{\Omega}$. In the one-dimensional case we used the interval $[-\pi, \pi]$ for our region $\Omega$, but could have used others as well provided they satisfy the conditions we specify later on. We will also assume $\Omega$ to be compact as in one dimension.

The first two conditions will guarantee that $\left\{S_{\Omega}(t-k)\right\}$ for $k \in \mathbf{Z}^{d}$ is an orthogonal basis of $V_{0}=\mathcal{B}_{\Omega}$. They are

(S1) $\Omega \cap\{\Omega+2 \pi k\} \simeq \varnothing$ for all $k \in \mathbf{Z}^{d}, k \neq 0$,

(S2) $\cup_{k \in \mathbf{Z}^{d}}\{\Omega+2 \pi k\} \simeq \mathbf{R}^{d}$.

The standard way (in wavelet theory) of showing orthogonality involves the following formula in the Fourier transform domain [1]:

$$
\sum_{k \in \mathbf{Z}^{d}}\left|\widehat{S}_{\Omega}(\omega+2 \pi k)\right|^{2}=1
$$

That this holds almost everywhere in our case is evident from these two conditions since $\widehat{S}_{\Omega}(\omega)=\chi_{\Omega}(\omega)$, the characteristic function of our region. We also get another result for free

$$
\sum_{k \in \mathbf{Z}^{d}} \widehat{S}_{\Omega}(\omega+2 \pi k)=1
$$

which again holds almost everywhere for $\omega \in \mathbf{R}^{d}$. This will enable us to get a sampling theorem for $f \in \mathcal{B}_{\Omega}$.

\section{Examples of sampling functions.}

- In one dimension, we have the interval $[-\pi, \pi]$ as our standard example, but any interval of length $2 \pi$ could have worked just as well. In fact, even more complex sets will work, e.g., $\Omega=[-\pi / 2, \pi / 2] \cup$ $[5 \pi / 2,7 \pi / 2]$. See $[\boldsymbol{5}]$ for more examples.

- In two dimensions, the Cartesian product of two of the one dimensional examples will work. Another example is $\Omega=D \cup(\{S-D+$ $(2 \pi, 0)\})$, where $D$ is the unit disk and $S=[-\pi, \pi]^{2}$.

- Another example in two dimensions with an interesting symmetry is the bow tie set. Let $\Xi_{1}$ be the set bounded by the lines $\omega_{1}+\omega_{2}=0$, $\omega_{1}-\omega_{2}=0, \omega_{2}=\pi, \omega_{2}=-\pi$ inside the square $S$ of $\Xi_{1}$, and let $\Xi_{2}$ 
be the complementary set obtained by replacing the last two lines by $\omega_{1}=\pi, \omega_{1}=-\pi$. Then $\Omega=\Xi_{1} \cup\left\{\Xi_{2}+(2 \pi, 0)\right\}$.

Clearly the function $S_{\Omega}(t)$ plays a number of roles in our space $\mathcal{B}_{\Omega}$. It is the reproducing kernel since $S_{\Omega} * f=f$, its integer translates are orthonormal $\left\langle S_{\Omega}, S_{\Omega}(\cdot-k)\right\rangle=\delta_{0 k}$, and it satisfies a sampling condition $S_{\Omega}(k)=\delta_{0 k}$. In fact, we can even say a little more.

Proposition 4. Let $\Omega$ be a set in $\mathbf{R}^{d}$ satisfying conditions (S1) and $(\mathrm{S} 2)$, let $S_{\Omega}(t)$ be the inverse Fourier transform of the characteristic function $\chi_{\Omega}(\omega)$. Then $\left\{S_{\Omega}(t-k)\right\}$ is an orthonormal basis of $\mathcal{B}_{\Omega}$ and

$$
f(t)=\sum_{k \in \mathbf{Z}^{d}} f(k) S_{\Omega}(t-k)
$$

for each $f \in \mathcal{B}_{\Omega}$, where the convergence is uniform and in the sense of $L^{2}\left(\mathbf{R}^{d}\right)$.

Although the proofs of these results are quite well known in the onedimensional case, we repeat them here for completeness.

(1) Orthogonality. By using (8) we get

$$
\begin{aligned}
\int_{\mathbf{R}^{d}} S_{\Omega}(t-k) & \overline{S_{\Omega}(t)} d t \\
& =(2 \pi)^{-d} \int_{\mathbf{R}^{d}}\left|\widehat{S}_{\Omega}(\omega)\right|^{2} e^{-i \omega \cdot k} d \omega \\
& =(2 \pi)^{-d} \sum_{n \in \mathbf{Z}^{d}} \int_{[-\pi, \pi]^{d}-2 \pi n}\left|\widehat{S}_{\Omega}(\omega)\right|^{2} e^{-i \omega \cdot k} d \omega \\
& =(2 \pi)^{-d} \int_{[-\pi, \pi]^{d}} \sum_{n \in \mathbf{Z}^{d}}\left|\widehat{S}_{\Omega}(\omega+2 \pi n)\right|^{2} e^{-i \omega \cdot k} d \omega \\
& =(2 \pi)^{-d} \int_{[-\pi, \pi]^{d}} e^{-i \omega \cdot k} d \omega=\delta_{0 k},
\end{aligned}
$$

which is the desired orthonormality. 
(2) Sampling condition. By using (9), we get

$$
\begin{aligned}
S_{\Omega}(k) & =(2 \pi)^{-d} \int_{\mathbf{R}^{d}} \widehat{S}_{\Omega}(\omega) e^{-i \omega \cdot k} d \omega \\
& =(2 \pi)^{-d} \int_{[-\pi, \pi]^{d}} \sum_{n \in \mathbf{Z}^{d}} \widehat{S}_{\Omega}(\omega+2 \pi n) e^{-i \omega \cdot k} d \omega \\
& =(2 \pi)^{-d} \int_{[-\pi, \pi]^{d}} e^{-i \omega \cdot k} d \omega=\delta_{0 k} .
\end{aligned}
$$

(3) Completeness. We assume $f \in \mathcal{B}_{\Omega}$ with all its coefficients equal to zero, i.e.,

$$
a_{k}=\int_{\mathbf{R}^{d}} f(t) \overline{S_{\Omega}(t-k)} d t=0, \quad k \in \mathbf{Z}^{d}
$$

then we must show that $f=0$. By using the Fourier transform, we see that these coefficients are given by

$$
\begin{aligned}
(2 \pi)^{-d} & \int_{\mathbf{R}^{d}} \widehat{f}(\omega) \widehat{S}_{\Omega}(\omega) e^{i \omega \cdot k} d \omega \\
& =(2 \pi)^{-d} \int_{[-\pi, \pi]^{d}} \sum_{n \in \mathbf{Z}^{d}} \widehat{f}(\omega+2 \pi n) \widehat{S}_{\Omega}(\omega+2 \pi n) e^{i \omega \cdot k} d \omega=0 .
\end{aligned}
$$

Since these are the Fourier coefficients of a periodic function, it follows that it must be zero as well,

$$
\begin{aligned}
\sum_{n \in \mathbf{Z}^{d}} \widehat{f}(\omega+2 \pi n) & \widehat{S}_{\Omega}(\omega+2 \pi n) \\
& =\sum_{n \in \mathbf{Z}^{d}} \widehat{f}(\omega+2 \pi n) \chi_{\Omega}(\omega+2 \pi n) \\
& =\widehat{f}(\omega) \chi_{\Omega}(\omega)=0 \quad \text { a.e. for } \omega \in \Omega
\end{aligned}
$$


(4) Uniform convergence. As with all orthonormal bases, we know the series expansion of $f$ converges in the sense of $L^{2}\left(\mathbf{R}^{d}\right)$. But in this case it also converges uniformly. Indeed we have that

$$
\begin{aligned}
& f(t)-\sum_{\substack{k \in \mathbf{Z}^{d} \\
k<N}} a_{k} S_{\Omega}(t-k) \\
& =(2 \pi)^{-d} \int_{\mathbf{R}^{d}}\left[\widehat{f}(\omega)-\sum_{k<N} a_{k} \widehat{S}_{\Omega}(\omega) e^{-i \omega \cdot k}\right] e^{i \omega \cdot t} d \omega \\
& =(2 \pi)^{-d} \int_{[-\pi, \pi]^{d}}\left[\sum_{n \in \mathbf{Z}^{d}} \widehat{f}(\omega+2 \pi n)-\sum_{k<N} a_{k} \widehat{S}_{\Omega}(\omega+2 \pi n) e^{-i \omega \cdot k}\right] e^{i \omega \cdot t} d \omega \\
& =(2 \pi)^{-d} \int_{[-\pi, \pi]^{d}}\left[\widehat{f}^{*}(\omega)-\sum_{k<N} a_{k} e^{-i \omega \cdot k}\right] e^{i \omega \cdot t} d \omega,
\end{aligned}
$$

where $\widehat{f}^{*}$ is the periodic extension of $\widehat{f}$. We then take absolute values to get

$$
\begin{aligned}
& \left|f(t)-\sum_{k \in \mathbf{Z}^{d}, k<N} a_{k} S_{\Omega}(t-k)\right| \\
& \quad \leq(2 \pi)^{-d}\left\{\int_{[-\pi, \pi]^{d}}\left|\widehat{f}^{*}(\omega)-\sum_{k<N} a_{k} e^{-i \omega \cdot k}\right|^{2} d \omega \int_{[-\pi, \pi]^{d}}\left|e^{i \omega \cdot t}\right|^{2} d \omega\right\}^{1 / 2} \\
& \quad=\left\|\widehat{f}^{*}-\sum_{k<N} a_{k} e^{-i \omega \cdot k}\right\|_{2} \rightarrow 0 \text { as } N \rightarrow \infty .
\end{aligned}
$$

since the $a_{k}$ are also the Fourier coefficients of $\widehat{f}^{*}$. The last bound is independent of $t$, and we have the required uniform convergence

(5) Sampling series. The coefficients are given by

$$
\begin{aligned}
a_{k} & =\int_{\mathbf{R}^{d}} f(t) \overline{S_{\Omega}(t-k)} d t \\
& =(2 \pi)^{-d} \int_{\mathbf{R}^{d}} \widehat{f}(\omega) \widehat{S}_{\Omega}(\omega) e^{i \omega \cdot k} d \omega \\
& =(2 \pi)^{-d} \int_{\Omega} \widehat{f}(\omega) e^{i \omega \cdot k} d \omega=f(k),
\end{aligned}
$$

and hence the uniform convergence holds for the sampling series as well. 
4. The PS wavelets. The wavelet system based on the PSWFs has several components (as do wavelets systems in general). These components include a scaling function $\phi$, whose $d$-dimensional integer translates form a basis of a space $V_{0}$, the mother wavelet $\psi$ and the multiresolution analysis $\left\{V_{m}\right\}$ of dilations of $V_{0}$. Both the scaling function and the mother wavelet are defined by PSWFs, but we shall concentrate on the former.

4.1 Scaling function. As in the one-dimensional case, we choose the scaling function of the PS wavelet to be the first PSWF, $\phi(t)=$ $\varphi_{0, \Omega, \Gamma}(t)$, and consider the set of translates of $\phi$ in $\mathbf{Z}^{d},\{\phi(t-n)\}_{n \in \mathbf{Z}^{d}}$. We assume that $\Omega$ satisfies conditions 1) and 2) needed to obtain a sampling function. If the concentration set $\Gamma$ is sufficiently large, the energy outside of the set will be negligible. This is clear if $\Gamma$ is the Cartesian product of intervals by the one-dimensional results. If we take "sufficiently large" to mean that $\Gamma$ contains a large $d$-dimensional interval centered at the origin, then the statement must be true in general as well.

We first have to show that $\{\phi(t-n)\}_{n \in \mathbf{Z}^{d}}$, if it is to be a bona fide scaling function, is a Riesz basis of $V_{0}=\mathcal{B}_{\Omega}$. Again this is clear if $\Gamma$ is a $d$-dimensional interval because in that case $\hat{\phi}$ is positive on $\Omega$. For the general case we add another condition

(W3) $\widehat{\varphi}_{0, \Omega, \Gamma}(\omega) \neq 0$ for $\omega \in \Omega$.

This is actually a condition on $\Gamma$ since we know that for some $\Gamma$ it does hold, but perhaps not for all. In the one-dimensional case it follows from the fact that $\phi$ is zero free in the interval $[-\tau, \tau]$ and is also the eigenfunction of the Fourier transform (with a change of scale).

Proposition 5. Let $\phi(t)=\varphi_{0, \Omega, \Gamma}(t)$, and let $\Omega$ and $\Gamma$ be such that conditions (S1), (S2) and (W3) hold. Then $\{\phi(t-n)\}_{n \in \mathbf{Z}^{d}}$ is a Riesz basis of $V_{0}$.

The proof is similar to that in one dimension and is most easily phrased in terms of a sufficient condition for a Riesz basis involving 
the Fourier transform. This condition is that if the series

$$
\sum_{n \in \mathbf{Z}^{d}}\left|\widehat{\varphi}_{0, \Omega, \Gamma}(\omega-2 \pi n)\right|^{2}
$$

has a positive upper and lower bound, then it is the Riesz basis of its close linear span [1]. Since $\widehat{\varphi}_{0, \Omega, \Gamma}$ has compact support on $\Omega$ and by condition (W3) is positive there, then it follows from condition (S1) that the lower bound is attained for some $\omega \in \Omega$ provided that $\widehat{\varphi}_{0, \Omega, \Gamma}$ is continuous on $\Omega$. To see that it is, we return to Proposition 3 , which gives us

$$
\begin{aligned}
\lambda_{0} \varphi_{0, \Omega, \Gamma}(x) & =\int_{\Gamma} S_{\Omega}(x-t) \varphi_{0, \Omega, \Gamma}(t) d t \\
& =\int_{\mathbf{R}^{d}} S_{\Omega}(x-t) \chi_{\Gamma}(t) \varphi_{0, \Omega, \Gamma}(t) d t \\
& =\left[S_{\Omega} *\left(\chi_{\Gamma} \varphi_{0, \Omega, \Gamma}\right)\right](x) .
\end{aligned}
$$

Since the Fourier transform takes convolutions into products, we find that

$$
\begin{aligned}
\lambda_{0} \widehat{\varphi}_{0, \Omega, \Gamma}(\omega) & =\lambda_{0} \widehat{\phi}(\omega)=\widehat{S_{\Omega}}(\omega)\left(\widehat{\chi_{\Gamma} \phi}\right)(\omega) \\
& =(2 \pi)^{d} \chi_{\Omega}(\omega)\left(\overline{S_{\Gamma}} * \widehat{\phi}\right)(\omega) \\
& =(2 \pi)^{d} \chi_{\Omega}(\omega) \int_{\Omega} \overline{S_{\Gamma}}(\omega-\xi) \widehat{\phi}(\xi) d \xi
\end{aligned}
$$

from which it follows by an elementary argument that $\widehat{\phi}$ is bounded and continuous on $\Omega$. The upper bound follows from the same sort of consideration.

We still have to show that the closed linear span of $\{\phi(t-n)\}$ is equal to $V_{0}$. Indeed, let $f \in V_{0}=\mathcal{B}_{\Omega}$, then $\widehat{f}$ has compact support on $\Omega$ and is in $L^{2}(\Omega)$ and so is $\widehat{f} / \widehat{\phi}$. Therefore it has a Fourier series

$$
\sum_{n} a_{n} e^{-i \omega \cdot n}
$$

convergent in the sense of $L^{2}(\Omega)$ to $\widehat{f} / \widehat{\phi}$, and

$$
\widehat{f}(\omega)=\sum_{n} a_{n} e^{-i \omega \cdot n} \widehat{\phi}(\omega) .
$$


By taking the inverse Fourier transform we get

$$
f(t)=\sum_{n} a_{n} \phi(t-n),
$$

which gives us our conclusion.

These formulae also give us a way of finding the coefficients in the scaling function series (11). Indeed the dual scaling function $\widetilde{\phi}$ is given by the well known construction in wavelet theory

$$
\widehat{\widetilde{\phi}}(\omega)=\frac{\overline{\widehat{\phi}(\omega)}}{\sum_{n \in \mathbf{Z}^{d}}|\widehat{\phi}(\omega-2 \pi n)|^{2}} .
$$

From this, it follows that

$$
a_{n}=\langle f, \widetilde{\phi}(\cdot-n)\rangle, \quad f \in \mathcal{B}_{\Omega} .
$$

Because of the localization property of wavelets, it is often possible to replace the integral in this last formula by $f(n) / \widehat{\phi}(0)$. The resulting series does not converge to $f$ but it will give a good approximation when we change scales in the next subsection.

4.2 Change of scale. In one dimension, a multiresolution analysis usually involves a scale change by a factor of two as was mentioned in Section 2. That is, the spaces $\left\{V_{m}\right\}$ are related to each other by the condition that $f \in V_{m}$ if and only if $f(2 \cdot) \in V_{m+1}$. In higher dimensions many other options are open to us - a dilation may still be by a factor of 2 , but can, in general, involve a linear transformation $A$ on $\mathbf{R}^{d}$ with certain properties. These are [5]:

(M4) $A$ is nonsingular and $A \mathbf{Z}^{d} \subset \mathbf{Z}^{d}$,

(M5) The eigenvalues of $A$ satisfy $\left|\lambda_{j}(A)\right|>1$.

The associated dilation subspaces $\left\{V_{m}\right\}$ then are related by $f(x) \in$ $V_{m}$ if and only if $f(A x) \in V_{m+1}$. We still need more conditions to ensure that $\left\{V_{m}\right\}$ is an MRA. These involve the relation between $A$ and the set $\Omega$ in the Fourier transformed space. Let $B=A^{*}$, the adjoint of $A$; we require that

(M6) $\Omega \subset B \Omega$ and $\cup_{m}\left(B^{m} \Omega\right) \simeq \mathbf{R}^{d}$. 
The following was proved by Madych [5]:

Proposition 6. Let $\Omega$ satisfy the conditions (S1) and (S2) in subsection 3.2, let A satisfy the conditions (M4) and (M5) above, let $\Omega, A$ satisfy condition (M6); then

(1) $V_{m}$ is the Paley-Wiener space of $L^{2}\left(\mathbf{R}^{d}\right)$ functions whose Fourier transforms vanish outside of $B^{m} \Omega$ and

(2) $\left\{V_{m}\right\}$ is a multiresolution analysis of $L^{2}\left(\mathbf{R}^{d}\right)$.

The corresponding Riesz basis of $V_{m}$ is given by $\left\{\phi\left(A^{m} x-n\right)\right\}$ provided condition (W3) is also satisfied. Hence every $f \in V_{m}$ has a convergent expansion in terms of this system. An alternate expansion, as mentioned in the previous subsection, is given by

$$
f_{m}(x)=\sum_{n}\left[f\left(A^{-m} n\right) / \widehat{\phi}(0)\right] \phi\left(A^{m} x-n\right) .
$$

While this series does not converge to $f(x)$ necessarily for fixed $m$, it does converge to $f_{m}(x)$, which then converges to it as $m \rightarrow \infty$. In fact, we have the same conclusion holding for a larger class of functions, those satisfying a certain differentiability condition independent of $m$. This differentiability condition is the one associated with Sobolev spaces $H^{\alpha}$, which, in turn, are characterized in terms of their Fourier transforms as $f \in H^{\alpha}$ whenever $\int|\widehat{f}(\omega)|^{2}\left(|\omega|^{2}+1\right)^{\alpha} d \omega<\infty[\mathbf{1 1}]$. This turns out to be a Hilbert space with the norm given by this expression and the obvious inner product. For $\alpha=1$ and $d=1$, it corresponds to functions which, together with their (weak) derivatives, belong to $L^{2}(\mathbf{R})$.

Proposition 7. Let $f \in H^{\alpha}\left(\mathbf{R}^{d}\right)$, let $f_{m} \in V_{m}$ be given by (12) and let $\alpha>\beta>d / 2$; then

$$
\left\|f_{m}-f\right\|_{\infty}=O\left((\operatorname{det} B)^{-m(\alpha-\beta)}\right) .
$$

Note that since the eigenvalues of $A$ and hence of $B$ are larger than 1 in magnitude, this difference must converge to 0 as $m \rightarrow \infty$. The proof of both is similar, and involves the Poisson summation formula applied to (12). We assume for the moment that $f=P^{m} f \in V_{m}\left(P^{m} f\right.$ is the 
projection of $f$ onto $V_{m}$ ) and compare its Fourier transform to that of $f_{m}$ which is

$$
\begin{aligned}
\widehat{f}_{m}(\omega) & =\sum_{n}\left[f\left(A^{-m} n\right) / \widehat{\phi}(0)\right] \widehat{\phi}\left(B^{-m} \omega\right) e^{-i B^{-m} \omega \cdot n}(\operatorname{det} A)^{-m} \\
& =\sum_{k}\left[\widehat{f}\left(\omega+2 \pi B^{m} k\right)\right] \widehat{\phi}\left(B^{-m} \omega\right) / \widehat{\phi}(0)
\end{aligned}
$$

Since both $\widehat{f}$ and $\widehat{f}_{m}$ have support in $B^{m} \Omega$, the difference between the two is merely

$$
\widehat{f}(\omega)-\widehat{f}_{m}(\omega)=\widehat{f}(\omega)\left[1-\widehat{\phi}\left(B^{-m} \omega\right) / \widehat{\phi}(0)\right]
$$

Supposing now that $f$ only satisfies the hypothesis, we look for a bound on the pointwise difference of $f$ and $f_{m}$. By the Fourier integral theorem we find that

$$
\begin{aligned}
\left|f_{m}(x)-f(x)\right| & \leq(2 \pi)^{-d}\left\|\widehat{f}_{m}-\widehat{f}\right\|_{1} \\
& \leq \frac{1}{(2 \pi)^{d}}\left(\left\|\widehat{f_{m}}-\widehat{P^{m} f}\right\|_{1}+\left\|\widehat{P^{m} f}-\widehat{f}\right\|_{1}\right) .
\end{aligned}
$$

We now apply (13) (with $f$ replaced by $P^{m} f$ ) to the first term on the right to get

$$
\begin{aligned}
(2 \pi)^{-d}|| \widehat{f}_{m}-\widehat{P^{m} f} \|_{1} \\
\quad \leq(2 \pi)^{-d}|| \widehat{P^{m} f} \|_{2}\left\{\int_{B^{m} \Omega}\left|\frac{\widehat{\phi}(0)-\widehat{\phi}\left(B^{-m} \omega\right)}{\widehat{\phi}(0)}\right|^{2} d \omega\right\}^{1 / 2} \\
\quad \leq(2 \pi)^{-d}\|\widehat{f}\|_{2}\left\{\int_{\Omega}\left|\frac{\widehat{\phi}(0)-\widehat{\phi}(\omega)}{\widehat{\phi}(0)}\right|^{2} d \omega\right\}^{1 / 2} \operatorname{det}(B)^{-m / 2}
\end{aligned}
$$

Since $\widehat{P^{m} f}$ and $\widehat{f}$ agree on $B^{m} \Omega$, and the former has support on this 
set, the second term obeys

$$
\begin{aligned}
& \frac{1}{(2 \pi)^{d}}\left\|\widehat{P^{m} f}-\widehat{f}\right\|_{1} \\
& =\frac{1}{(2 \pi)^{d}} \int_{\mathbf{R}^{d}-B^{m} \Omega}|\widehat{f}(\omega)| d \omega \\
& =\frac{1}{(2 \pi)^{d}} \int_{\mathbf{R}^{d}-B^{m} \Omega}|\widehat{f}(\omega)|\left(|\omega|^{2}+1\right)^{\alpha / 2}\left(|\omega|^{2}+1\right)^{-\alpha / 2} d \omega \\
& \leq \frac{1}{(2 \pi)^{d}}\left\{\int_{\mathbf{R}^{d}}|\widehat{f}(\omega)|^{2}\left(|\omega|^{2}+1\right)^{\alpha} d \omega \int_{\mathbf{R}^{d}-B^{m} \Omega}\left(|\omega|^{2}+1\right)^{-\alpha} d \omega\right\}^{1 / 2} \\
& =\frac{1}{(2 \pi)^{d}}\|f\|_{H^{\alpha}}\left\{\int_{\mathbf{R}^{d}-B^{m} \Omega}\left(|\omega|^{2}+1\right)^{-\alpha} d \omega\right\}^{1 / 2} .
\end{aligned}
$$

Here $\|f\|_{H^{\alpha}}$ denotes the norm of $f$ in the Sobolev space. We finish off the proof by observing that

$$
\begin{aligned}
\int_{\mathbf{R}^{d}-B^{m} \Omega}\left(|\omega|^{2}\right. & +1)^{-\alpha} d \omega \\
& \leq \sup _{\mathbf{R}^{d}-B^{m} \Omega}\left(|\omega|^{2}+1\right)^{-\alpha+\beta} \int_{\mathbf{R}^{d}-B^{m} \Omega}\left(|\omega|^{2}+1\right)^{-\beta} d \omega
\end{aligned}
$$

and that $\omega \notin B^{m} \Omega$ implies that $B^{-m} \omega \notin \Omega$. Since $\Omega$ contains a neighborhood of 0 , it follows that $\left|B^{-m} \omega\right|>c>0$. Hence, we find that

$$
\sup _{\mathbf{R}^{d}-B^{m} \Omega}\left(|\omega|^{2}+1\right)^{-\alpha+\beta}<(\operatorname{det} B)^{-2 m(\alpha-\beta)} c,
$$

which gives us our conclusion.

The most commonly used case is that of dyadic dilation in which $A=B=2 I$. Then $\operatorname{det} B=2^{d}$; but in all cases we have $\operatorname{det} B \geq 2$, by the conditions on the eigenvalues. Hence we always get uniform convergence.

4.3 Mother wavelet. The mother wavelet can be defined in a number of different ways. It should be orthogonal to $V_{0}$ and belong to $V_{1}$; it should be a basis of the orthogonal complement $W_{0}$ of $V_{0}$ in $V_{1}$. It is even possible to make it an orthogonal basis. The orthogonality property of $W_{0}$ is easy to come by since any function whose Fourier 
transform has support in the set $\overline{B \Omega-\Omega}$ will be orthogonal to $V_{0}$ and belongs to $V_{1}$.

In the one-dimensional case we used the a pair of PSWFs, one of which had support in $[-2 \pi,-\pi]$ and the other in $[\pi, 2 \pi]$. Then $\widehat{\psi}(\omega):=\left(\widehat{\varphi}_{0, \pi / 2, \tau}(\omega-3 \pi / 2)+\widehat{\varphi}_{0, \pi / 2, \tau}(\omega+3 \pi / 2)\right) / 2$, and it was shown that translates of $\psi$ constituted a Riesz basis of $W_{0}$.

In the two-dimensional case where $\Omega$ is a square and $B=2 I$, we can split the set $2 \Omega-\Omega$ into eight congruent squares and find the shifted Fourier transform of a PSWF on each of the squares. This is then combined into the Fourier transform of the mother wavelet. Since this is just a repetition of the arguments in the one-dimensional case, we shall not pursue it further. In fact, since we are primarily concerned with approximation rather than with decomposition, we shall not use the mother wavelet in the remainder of this work.

4.4 Computation of PS scaling functions. The maximization problem of Section 3 consists of maximizing the ratio

$$
\rho=\int_{\Gamma}|f(t)|^{2} d t / \int_{\mathbf{R}^{d}}|f(t)|^{2} d t
$$

for functions $f$ whose Fourier transforms have support on $\Omega$. Such functions may be represented by Proposition 4 as

$$
f(t)=\sum_{n \in \mathbf{Z}^{d}} f(n) S_{\Omega}(t-n),
$$

where $S_{\Omega}(t)$ is the generalization of the sinc function. This sequence of functions $\left\{S_{\Omega}(t-n)\right\}$ is also an orthonormal basis of $B_{\Omega}$ and hence the coefficients $\{f(n)\} \in l^{2}\left(\mathbf{Z}^{d}\right)$. We then can substitute this series into both integrals in (16) to get, after an interchange of integrals and summations,

$$
\sum_{n} f(n) \sum_{k} \overline{f(k)} \int_{\Gamma} S_{\Omega}(t-n) \overline{S_{\Omega}(t-k)} d t
$$

in the numerator and

$$
\sum_{n} f(n) \sum_{k} \overline{f(k)} \int_{\mathbf{R}^{d}} S_{\Omega}(t-n) \overline{S_{\Omega}(t-k)} d t=\sum_{n}|f(n)|^{2}
$$


in the denominator. The former is valid because of the dominated convergence theorem, while the latter is a result of Parseval's equality.

We now denote by $A_{\Gamma, \Omega}$ the function on $\mathbf{Z}^{2 d}$ given by

$$
A_{\Gamma, \Omega}(n, k)=\int_{\Gamma} S_{\Omega}(t-n) \overline{S_{\Omega}(t-k)} d t .
$$

Thus the ratio in (16) can be expressed as

$$
\rho=\frac{\left\langle\mathbf{f}, A_{\Gamma, \Omega} \mathbf{f}\right\rangle}{\langle\mathbf{f}, \mathbf{f}\rangle}
$$

where $\mathbf{f}$ now denotes the multi-sequence $\{f(n)\}$ and the inner product is just the $l^{2}\left(\mathbf{Z}^{d}\right)$ inner product. This may be considered as an operator on $l^{2}\left(\mathbf{Z}^{d}\right)$ which again is nonnegative and self-adjoint. In fact, it is positive definite since the inner product in the numerator of (18) is

$$
\left\langle\mathbf{f}, A_{\Gamma, \Omega} \mathbf{f}\right\rangle=\int_{\Gamma}|f(t)|^{2} d t
$$

and cannot be zero for nonzero $f$, as discussed in Section 3. The operator is also compact by the same consideration as the operator $S_{\Omega, \Gamma}$ on $\mathcal{B}_{\Omega}$.

Such operators have eigenvalue, eigenfunction pairs in which the eigenvalues are all positive and between 0 and 1 . In fact these eigenvalues are identical to those of $S_{\Omega, \Gamma}$ because of the equivalence of (16) and (18).

The problem of maximizing the quotient in (18) is solved by finding the maximum eigenvalue of the operator $A_{\Gamma, \Omega}$ and its associated eigenfunction. This is carried out by first truncating $A_{\Gamma, \Omega}(n, k)$ to some bounded set in $\mathbf{Z}^{2 d}$ and then ordering the $d$-tuples $n$ and $k$ in some convenient way. This converts the operator into a matrix $A$ whose eigenvalues and eigenvectors approximate those of $A_{\Gamma, \Omega}$. The finite sequence $\vec{\phi}=\left\{\phi_{n}\right\}$ and number $\lambda$ such that $A \vec{\phi}=\lambda \vec{\phi}$ where $\lambda$ is maximum value of the ratio is used to approximate the solution to (18). This then is used to solve the original maximization problem by using the associated sampling theorem.

$$
\phi(t)=\sum_{n} \phi_{n} S_{\Omega}(t-n) .
$$


The function $\phi(t)$ is the scaling function for the PS wavelets and is the first prolate spheroidal wave function.

We may extend this result to other eigenvalues and eigenvectors but do not do so since we are interested only in the dominant one for our PS wavelet. The calculations just described have been carried out for certain cases in which the eigenvalues and eigenvectors were found by MAPLE. The results were promising, but no systematic error analysis has as yet been carried out.

\section{REFERENCES}

1. I. Daubechies, Ten lectures on wavelets, SIAM, Philadelphia, 1992.

2. H.J. Landau and H.O. Pollak, Prolate spheroidal wave functions, Fourier analysis and uncertainty, II, Bell System Tech. J. 40 (1961), 65-84.

3. - Prolate spheroidal wave functions, Fourier analysis and uncertainty, III, Bell System Tech. J. 41 (1962), 1295-1336.

4. H.J. Landau and H. Widom, Eigenvalue distribution of time and frequency limiting, J. Math. Anal. Appl. 77 (1980), 469-481.

5. W.R. Madych, Some elementary properties of multiresolution analysis of $L^{2}\left(\mathbf{R}^{n}\right)$, in Wavelets - A tutorial in theory and applications (C. Chui, ed.), Academic Press, Boston, 1992.

6. A. Papoulis, Signal analysis, McGraw Hill, New York, 1977.

7. D. Slepian, Some comments on Fourier analysis, uncertainty, and modeling, SIAM Review 25 (1983), 379-393.

8. - Prolate spheroidal wave functions, Fourier analysis and uncertainty, IV, Bell System Tech. J. 43 (1964), 3009-3058.

9. D. Slepian and H.O. Pollak, Prolate spheroidal wave functions, Fourier analysis and uncertainty, I, Bell System Tech. J. 40 (1961), 43-64.

10. T. Soleski and G.G. Walter, A new friendly method of computing prolate spheroidal wave functions and wavelets, Appl. Comp. Harmonic Anal. 19 (2005), 432-443.

11. I. Stakgold, Green's functions and boundary value problems, John Wiley and Sons, New York, 1979.

12. G. Szego, Orthogonal polynomials, AMS Colloq. Publ. 23, Amer. Math. Soc., Providence, 1974.

13. G.G. Walter, Prolate spheroidal wavelets: Differentiation, translation, and convolution made easy, J. Fourier Anal. Appl. 11 (2005), 73-84.

14. G.G. Walter and X. Shen, Wavelets based on prolate spheroidal wave functions, J. Fourier Anal. Appl. 10 (2004), 1-25. 
15. — Sampling with prolate spheroidal functions, J. Sampling Theory Signal Image Processing 2 (2003), 25-52.

16. G.G. Walter and X. Shen, Wavelets and other orthogonal systems, 2nd ed., CRC Press, Boca Raton, Florida, 2001.

17. A. Zayed, Advances in Shannon's sampling theory, CRC Press, Boca Raton, Florida, 1993.

18. S. Zhang and J.M. Jin, Computation of special functions, Wiley, New York, 1996.

19. S. Zygmund, Trigonometric series, Cambridge, 1959.

Department of Mathematical Sciences, University of WisconsinMilwaukee, Milwaukee, WI 53201

E-mail address: ggw@uwm.edu 